\title{
Baxter Permutations, Maj-balances, and Positive Braids
}

\author{
Sen-Peng Eu ${ }^{a, b} \quad$ Tung-Shan $\mathrm{Fu}^{c} \quad$ Yeh-Jong Pan ${ }^{d}$ Chien-Tai Ting ${ }^{a, b, *}$ \\ ${ }^{a}$ Dept of Applied Mathematics, National University of Kaohsiung \\ Kaohsiung 811, Taiwan, ROC \\ ${ }^{b}$ Dept of Mathematics and Physics, Air Force Academy \\ Kaohsiung 820, Taiwan, ROC \\ ${ }^{c}$ Mathematics Faculty, National Pingtung Institute of Commerce \\ Pingtung 900, Taiwan, ROC \\ ${ }^{d}$ Dept of Computer Science and Information Engineering \\ Tajen University, Pingtung 907, Taiwan, ROC
}

Submitted: Nov 27, 2011; Accepted: Aug 17, 2012; Published: Aug 30, 2012

Mathematics Subject Classifications: 05A05, 05A15, 05A19

\begin{abstract}
In this paper we present two interesting properties of 321-avoiding Baxter permutations. The first one is a variant of refined major-balance identity for the 321avoiding Baxter permutations, respecting the number of fixed points and descents. The second one is a bijection between the 321-avoiding Baxter permutations with the entry 1 preceding the entry 2 and the positive braid words on four strands.
\end{abstract}

Keywords: Baxter permutation, 321-avoiding, 123-avoiding, maj-balance, positive braid word, generating tree

\section{Introduction}

\subsection{Baxter permutations and Pattern avoidance.}

Let $\mathfrak{S}_{n}$ be the set of all permutations of $\{1, \cdots, n\}$. A permutation $\pi \in \mathfrak{S}_{n}$ is called a Baxter permutation if it satisfies the following conditions for all $1 \leqslant a<b<c<d \leqslant n$,

- if $\pi_{a}+1=\pi_{d}$ and $\pi_{b}>\pi_{d}$ then $\pi_{c}>\pi_{d}$;

\footnotetext{
*E-mail address: speu@nuk.edu.tw (S.-P. Eu), tsfu@npic.edu.tw (T.-S. Fu), yjpan@mail.tajen.edu.tw (Y.-J. Pan), d0994101@mail.nuk.edu.tw, chientaiting@gmail.com (C.-T. Ting).
} 
- if $\pi_{d}+1=\pi_{a}$ and $\pi_{c}>\pi_{a}$ then $\pi_{b}>\pi_{a}$.

For example, 25314 is a Baxter permutation, but 5327146 is not. It is clear from the definition that the reverse of a Baxter permutation is also Baxter. Let $\mathfrak{B}_{n}$ be the set of Baxter permutations in $\mathfrak{S}_{n}$. Chung, Graham, Hoggatt, and Kleiman [4] first proved analytically that

$$
\left|\mathfrak{B}_{n}\right|=\frac{2}{n(n+1)^{2}} \sum_{k=1}^{n}\left(\begin{array}{c}
n+1 \\
k-1
\end{array}\right)\left(\begin{array}{c}
n+1 \\
k
\end{array}\right)\left(\begin{array}{c}
n+1 \\
k+1
\end{array}\right) .
$$

A bijective proof is given by Viennot [17]. Other proofs are given by Mallows [10], Dulucq and Guibert [6]. Recently, Felsner, Fusy, Noy, and Orden [8] established explicit bijections between a number of objects that are enumerated by Baxter numbers.

For permutations $\pi=\pi_{1} \cdots \pi_{n} \in \mathfrak{S}_{n}$ and $\omega=\omega_{1} \cdots \omega_{t} \in \mathfrak{S}_{t}, t \leqslant n$, we say that $\pi$ contains an $\omega$-pattern if there are indices $i_{1}<\cdots<i_{t}$ such that $\pi_{i_{j}}<\pi_{i_{k}}$ if and only if $\omega_{j}<\omega_{k}$. Moreover, $\pi$ is called $\omega$-avoiding if $\pi$ contains no $\omega$-patterns. Let $\mathfrak{S}_{n}(\omega)$ denote

the set of $\omega$-avoiding permutations in $\mathfrak{S}_{n}$. It is known that $\left|\mathfrak{S}_{n}(\omega)\right|=C_{n}=\frac{1}{n+1}\left(\begin{array}{c}2 n \\ n\end{array}\right)$, the $n$th Catalan number, for every $\omega \in \mathfrak{S}_{3}$.

Let $\mathfrak{B}_{n}(\omega)$ be the set of $\omega$-avoiding permutations in $\mathfrak{B}_{n}$. Mansour and Vajnovszki [12] enumerated the permutations in $\mathfrak{B}_{n}(123)$ that avoid (or contain) another pattern of a certain length. They proved that the generating function for $\left|\mathfrak{B}_{n}(123)\right|$ is given by

$$
\frac{1-2 z+z^{2}}{1-3 z+2 z^{2}-z^{3}}=1+z+2 z^{2}+5 z^{3}+12 z^{4}+28 z^{5}+65 z^{6}+\cdots .
$$

They also pointed out that $\left|\mathfrak{B}_{n}(123)\right|=p_{3 n+3}$, where $\left\{p_{n}\right\}_{n \geqslant 0}$ is the Padovan sequence, defined by $p_{n}=p_{n-2}+p_{n-3}$ with $p_{0}=1, p_{1}=p_{2}=0$. Note that $\left|\mathfrak{B}_{n}(321)\right|=\left|\mathfrak{B}_{n}(123)\right|$ and $\mathfrak{B}_{n}(\omega)=\mathfrak{S}_{n}(\omega)$, for every $\omega \in\{132,213,231,312\}$.

\subsection{Refined sign-balance identities.}

In addition to the enumeration of $\mathfrak{S}_{n}(\omega)$ for $\omega \in \mathfrak{S}_{3}$, Simion and Schmidt [14] determined the sign-balance of the set $\mathfrak{S}_{n}(321)$

$$
\sum_{\pi \in \mathfrak{S}_{n}(321)}(-1)^{\operatorname{inv}(\pi)}=\left\{\begin{array}{cl}
C_{\frac{n-1}{2}} & \text { if } n \text { odd } \\
0 & \text { if } n \text { even }
\end{array}\right.
$$

where $\operatorname{inv}(\pi)=\left|\left\{\left(\pi_{i}, \pi_{j}\right): \pi_{i}>\pi_{j}, 1 \leqslant i<j \leqslant n\right\}\right|$ is the inversion number of $\pi$. Making use of a multivariate generating function, Adin and Roichman [1] proved a refinement of Eq. (1) with respect to the statistic $\operatorname{Ides}(\pi)$, the last descent, of $\pi \in \mathfrak{S}_{n}(321)$, i.e., $\operatorname{Ides}(\pi)=\max \left\{i: \pi_{i}>\pi_{i+1}, 1 \leqslant i \leqslant n-1\right\}$.

Theorem 1.1 (Adin-Roichman) The following identities hold. 


$$
\begin{aligned}
\sum_{\pi \in \mathfrak{S}_{2 n+1}(321)}(-1)^{i n v(\pi)} q^{\operatorname{ldes}(\pi)} & =\sum_{\pi \in \mathfrak{S}_{n}(321)} q^{2 \cdot \operatorname{ldes}(\pi)} \quad(n \geqslant 0), \\
\sum_{\pi \in \mathfrak{S}_{2 n}(321)}(-1)^{i n v(\pi)} q^{\operatorname{ldes}(\pi)} & =(1-q) \sum_{\pi \in \mathfrak{S}_{n}(321)} q^{2 \cdot \operatorname{ldes}(\pi)} \quad(n \geqslant 1) .
\end{aligned}
$$

At the same time, Mansour [11] found variation of the identities for $\mathfrak{S}_{n}(132)$, and shortly afterward, Reifegerste [13] proved analogous identities combinatorially respecting the length of the longest increasing subsequence of $\pi \in \mathfrak{S}_{n}(321)$. Recently, the authors obtained an analogous result for 321-avoiding alternating permutations, respecting the first (resp. last) entry of the permutations [7].

These results reveal a phenomenon that the signed enumerator of objects of size $2 n$ is essentially equal to the ordinary enumerator of objects of size $n$. One would naturally ask if there is any similar identity on other classes of (restricted) permutations, but computer checks show that this phenomenon seldom appears.

In the first part of this paper we will present a variant of refined balance identities for 321-avoiding Baxter permutations, where the sign of a permutation depends on the parity of its major index. To wit, we are considering the refined 'maj-balance' of $\mathfrak{B}_{n}(321)$. Note that the notion of maj-balance was also mentioned in [16].

We consider the following statistics of $\pi=\pi_{1} \cdots \pi_{n} \in \mathfrak{S}_{n}$. Let fix $(\pi)$ be the number of fixed points of $\pi$, i.e., fix $(\pi)=\left|\left\{i: \pi_{i}=i, 1 \leqslant i \leqslant n\right\}\right|$. The descent set of $\pi$ is defined as $\operatorname{Des}(\pi)=\left\{i: \pi_{i}>\pi_{i+1}, 1 \leqslant i \leqslant n-1\right\}$, and the descent number (des) and major $\operatorname{index}(\operatorname{maj})$ of $\pi$ are defined by $\operatorname{des}(\pi)=|\operatorname{Des}(\pi)|$ and $\operatorname{maj}(\pi)=\sum_{i \in \operatorname{Des}(\pi)} i$. The statistics maj and des play an important role in combinatorics. For example, the celebrated Carlitz identity [3]

$$
\frac{\sum_{\pi \in \mathfrak{S}_{n}} t^{\operatorname{maj}(\pi)} q^{\operatorname{des}(\pi)}}{\prod_{i=0}^{n}\left(1-q t^{i}\right)}=\sum_{k \geqslant 0}\left(1+t+\cdots+t^{k}\right)^{n} q^{n}
$$

has stimulated a lot of work.

Our first main result is the following maj-fix-des version of refined maj-balance identity for $\mathfrak{B}_{n}(321)$.

Theorem 1.2 For $n \geqslant 0$, we have

$$
\sum_{\pi \in \mathfrak{B}_{2 n+1}(321)}(-1)^{\operatorname{maj}(\pi)} p^{f i x(\pi)} q^{\operatorname{des}(\pi)}=p \cdot \sum_{\pi \in \mathfrak{B}_{n}(321)} p^{2 \cdot f i x(\pi)} q^{2 \cdot \operatorname{des}(\pi)} .
$$

We will prove this result via recurrence relation of multivariate generating functions. For the case of even length, we show that the maj-signed fix-des-enumerator of $\mathfrak{B}_{2 n}(321)$ can be expressed as a combination of the ordinary fix-des-enumerators of $\mathfrak{B}_{i}(321)$ for all $i=0,1, \ldots, n$. (See Theorem 2.5.) Moreover, we will also obtain a counterpart result on $\mathfrak{B}_{2 n+1}(123)$. (See Corollary 3.1) 


\subsection{Positive braid words.}

The Braid group on $n$ strands is the group generated by $\left\{\sigma_{1}, \cdots, \sigma_{n-1}\right\}$ with the relations

$$
\begin{cases}\sigma_{i} \sigma_{j}=\sigma_{j} \sigma_{i} & \text { for }|i-j| \geqslant 2 \\ \sigma_{i} \sigma_{j} \sigma_{i}=\sigma_{j} \sigma_{i} \sigma_{j} & \text { for }|i-j|=1\end{cases}
$$

Note that if we add the relation $\sigma_{i}^{2}=e$, then we get a presentation of the symmetric group $\mathfrak{S}_{n}$ on $n$ elements. A braid word is a finite sequence of letters $\sigma_{i}$ and their inverses $\sigma_{i}^{-1}$. A positive braid word is a braid word that contains no letter $\sigma_{i}^{-1}$. The set of positive braid words is also known as the monoid of positive braids, with concatenation as multiplication. For positive braid words, the length is an invariant, which is equal to the number of their letters. We refer the readers to [9] for more information.

To our surprise, by a search in OEIS [15], the generating function for $\left|\mathfrak{B}_{n}(123)\right|=$ $\left|\mathfrak{B}_{n}(321)\right|$ is very close to the one for the number of positive braid words on four strands (i.e., with letters $\left.\left\{\sigma_{1}, \sigma_{2}, \sigma_{3}\right\}\right)$. It has appeared in [15, A097550] that the generating function for the number of such words of length $n$ is given by

$$
\frac{1+z^{2}}{1-3 z+2 z^{2}-z^{3}}=1+3 z+8 z^{2}+19 z^{3}+44 z^{4}+102 z^{5}+237 z^{6}+\cdots .
$$

Note that this rational function has exactly the same denominator as the generating function for $\left|\mathfrak{B}_{n}(123)\right|=\left|\mathfrak{B}_{n}(321)\right|$.

Back to 321-avoiding Baxter permutations, our second main result is to establish a connection between the 321-avoiding Baxter permutations with the entry 1 preceding the entry 2 and the positive braid words on four strands.

Theorem 1.3 There is a bijection between the permutations $\pi \in \mathfrak{B}_{n+2}(321)$ with the condition $\pi^{-1}(1)<\pi^{-1}(2)$ and the positive braid words on four strands of length $n$.

To prove this theorem, we will construct generating trees for both families and show that they share the same succession rules. Readers are referred to $[5,18]$ for more information on generating trees.

The rest of the paper is organized as follows. In Section 2 we will first deal with $\mathfrak{B}_{2 n+1}(321)$ and prove Theorem 1.2 , then derive a similar identity on $\mathfrak{B}_{2 n}(321)$. In Section 3 we will obtain a counterpart result on $\mathfrak{B}_{2 n+1}(123)$. In Section 4 we will prove Theorem 1.3.

\section{Recurrence relation of multivariate generating functions for $\mathfrak{B}_{n}(321)$}

The definition of Baxter permutation seems complicated. As Dulucq and Guibert [6] put it in a perceivable way (see also [2]), it is equivalent to say that a Baxter permutation $\pi$ can be written as either of the two forms 
- $\pi=\left(\pi^{\prime}, i, \omega_{-}, \omega_{+}, i+1, \pi^{\prime \prime}\right)$,

- $\pi=\left(\pi^{\prime}, i+1, \omega_{+}, \omega_{-}, i, \pi^{\prime \prime}\right)$,

where all entries occurring in $\omega_{+}$(resp. $\omega_{-}$) are larger (resp. smaller) than $i$.

In this section, we shall prove Theorem 1.2 by making use of recurrence relations of multivariate generating functions. This approach starts from an inductive construction for 321-avoiding Baxter permutations. In fact, it is the reverse of a construction for 123avoiding Baxter permutations, given by Mansour and Vajnovszki [12]. A proof is included below for completeness.

Proposition 2.1 Let $\pi \in \mathfrak{B}_{n}(321)$, then either

(i) $\pi=\left(\pi^{\prime}, n\right)$ where $\pi^{\prime} \in \mathfrak{B}_{n-1}(321)$, or

(ii) there exist $1 \leqslant j \leqslant i \leqslant n-1$ such that $\pi=\left(\pi^{\prime}, i+1, i+2, \ldots, n, j, j+1, \ldots, i\right)$, where $\pi^{\prime} \in \mathfrak{B}_{j-1}(321)$.

Proof: Given a permutation $\pi=\pi_{1} \cdots \pi_{n} \in \mathfrak{B}_{n}(321)$, if $\pi_{n}=n$ then the proposition holds immediately by definition. Suppose $\pi_{n}=i(1 \leqslant i \leqslant n-1)$, let $\pi_{d}=n$. Since $\pi$ is 321-avoiding, the permutation $\pi$ contains the subsequence $(i+1, i+2, \cdots, n)$ and $\pi_{d+1}<\cdots<\pi_{n}$. Moreover, since $\pi$ is Baxter, the permutation $\pi$ is of the form $\pi=$ $\left(\pi^{\prime}, i+1, i+2, \ldots, n, \pi_{d+1}, \cdots, \pi_{n}\right)$. We observe that $\left(\pi_{d+1}, \cdots, \pi_{n}\right)=(j, j+1, \cdots, i)$, where $j=d+i+1-n$; otherwise there are entries $\ell$ and $k$ with $\ell<j \leqslant k$ such that $k \in \pi^{\prime}$ and $\ell, k+1 \in\left\{\pi_{d+1}, \cdots, \pi_{n}\right\}$, which is against the definition of Baxter permutations. Hence the second case holds.

Let us define the generating function for 321-avoiding Baxter permutations, respecting the statistics maj, fix, and des, by

$$
b_{n}:=b_{n}(t, p, q)=\sum_{\pi \in \mathfrak{B}_{n}(321)} t^{\operatorname{maj}(\pi)} p^{\mathrm{fix}(\pi)} q^{\operatorname{des}(\pi)},
$$

and let $B(t, p, q, z)=\sum_{n \geqslant o} b_{n}(t, p, q) z^{n}$. The initial terms of $B(t, p, q, z)$ are

$$
\begin{aligned}
B(t, p, q, z)=1 & +p z+\left(p^{2}+t q\right) z^{2}+\left[p^{3}+\left(t+t^{2}+\left(t+t^{2}\right) p\right) q\right] z^{3} \\
& +\left[p^{4}+\left(t+t^{2}+t^{3}+\left(t+2 t^{2}+t^{3}\right) p+\left(t+t^{2}+t^{3}\right) p^{2}\right) q+t^{4} q^{2}\right] z^{4} \\
& +\left[p^{5}+\left(t+t^{2}+t^{3}+t^{4}+\left(t+2 t^{2}+2 t^{3}+t^{4}\right) p+\left(t+2 t^{2}+2 t^{3}+t^{4}\right) p^{2}\right.\right. \\
& \left.\left.+\left(t+t^{2}+t^{3}+t^{4}\right) p^{3}\right) q+\left(t^{4}+2 t^{5}+t^{6}+\left(t^{4}+t^{5}+t^{6}\right) p\right) q^{2}\right] z^{5}+\cdots
\end{aligned}
$$

Theorem 1.2 can be rephrased as the identity

$$
b_{2 n+1}(-1, p, q)=p \cdot b_{n}\left(1, p^{2}, q^{2}\right)
$$


For example, for $n=2, b_{2}(t, p, q)=p^{2}+t q$. When $t=1$, we have $b_{2}(1, p, q)=p^{2}+q$. Take

$$
\begin{gathered}
b_{5}(t, p, q)=p^{5}+\left[t+t^{2}+t^{3}+t^{4}+\left(t+2 t^{2}+2 t^{3}+t^{4}\right) p+\left(t+2 t^{2}+2 t^{3}+t^{4}\right) p^{2}\right. \\
\left.+\left(t+t^{2}+t^{3}+t^{4}\right) p^{3}\right] q+\left[t^{4}+2 t^{5}+t^{6}+\left(t^{4}+t^{5}+t^{6}\right) p\right] q^{2} .
\end{gathered}
$$

When $t=-1$, we have $b_{5}(-1, p, q)=p^{5}+p q^{2}=p \cdot b_{2}\left(1, p^{2}, q^{2}\right)$. We derive a recurrence relation for $b_{n}(t, p, q)$ from Proposition 2.1.

Lemma 2.2 The sequence $\left\{b_{n}\right\}_{n \geqslant 0}$ satisfies the following recurrence relation

$$
b_{n}=(1+t+p) b_{n-1}-\left(t+p+t p-t^{n-1} q\right) b_{n-2}+t p \cdot b_{n-3},
$$

with $b_{0}=1, b_{1}=p$, and $b_{2}=p^{2}+t q$.

Proof: We construct the generating function $b_{n}$ for $\mathfrak{B}_{n}(321)$ by Proposition 2.1. The first case contributes $p \cdot b_{n-1}$ since $\pi_{n}=n$ is a fixed point, and the second case contributes $t^{n-i+j-1} q \cdot b_{j-1}$, for all $1 \leqslant j \leqslant i \leqslant n-1$, since $\pi_{n-i+j-1}=n$ contributes a descent. Therefore, we have

$$
b_{n}=p \cdot b_{n-1}+\sum_{i=1}^{n-1} \sum_{j=1}^{i} t^{n-i+j-1} q \cdot b_{j-1},
$$

where $b_{0}=1, b_{1}=p$ and $b_{2}=p^{2}+t q$. Consider Eq. (6) as a function $f(n)$ in $n$. Computing $f(n)-t \cdot f(n-1)$ leads to

$$
b_{n}-t \cdot b_{n-1}=p \cdot b_{n-1}-t p \cdot b_{n-2}+\sum_{j=1}^{n-1} t^{j} q \cdot b_{j-1} .
$$

By iteration, it follows from $f(n-1)-t \cdot f(n-2)$ that

$$
b_{n-1}-t \cdot b_{n-2}=p \cdot b_{n-2}-t p \cdot b_{n-3}+\sum_{j=1}^{n-2} t^{j} q \cdot b_{j-1} .
$$

Then the assertion follows from subtracting Eq. (8) from Eq. (7).

When $t=1$, we can derive a closed form for the fix-des enumerator of $\mathfrak{B}_{n}(321)$ from the recurrence relation. Let $g_{n}:=g_{n}(p, q)=b_{n}(1, p, q)$, i.e., $g_{n}(p, q)=\sum_{\pi \in \mathfrak{B}_{n}(321)} p^{\mathrm{fix}(\pi)} q^{\operatorname{des}(\pi)}$. It follows from Lemma 2.2 that

$$
g_{n}=(2+p) g_{n-1}-(1+2 p-q) g_{n-2}+p \cdot g_{n-3},
$$

with $g_{0}=1, g_{1}=p$ and $g_{2}=p^{2}+q$. Let $G:=G(p, q, z)=B(1, p, q, z)$, i.e., $G=$ $\sum_{n \geqslant 0} g_{n} z^{n}$. 
Corollary 2.3 The generating function $G(p, q, z)$ for the number of 321-avoiding Baxter permutations in $\mathfrak{S}_{n}$, respecting the number of fixed points and the number of descents, is given by

$$
G(p, q, z)=\frac{1-2 z+z^{2}}{1-(2+p) z+(1+2 p-q) z^{2}-p z^{3}}
$$

Proof: Taking a summation $\sum_{n \geqslant 3} g_{n} z^{n}$ on both sides of Eq. (9), we have

$$
G-1-p z-\left(p^{2}+q\right) z^{2}=(2+p) z(G-1-p z)-(1+2 p-q) z^{2}(G-1)+p z^{3} G .
$$

The assertion follows.

When $t=-1$, we consider the maj-signed fix-des enumerators of $\mathfrak{B}_{n}(321)$. Let $h_{n}:=$ $h_{n}(p, q)=b_{n}(-1, p, q)$, i.e., $h_{n}(p, q)=\sum_{\pi \in \mathfrak{B}_{n}(321)}(-1)^{\operatorname{maj}(\pi)} p^{\mathrm{fix}(\pi)} q^{\operatorname{des}(\pi)}$. It follows from Lemma 2.2 that

$$
h_{n}=p \cdot h_{n-1}+\left(1-(-1)^{n} q\right) h_{n-2}-p \cdot h_{n-3},
$$

with $h_{0}=1, h_{1}=p$ and $h_{2}=p^{2}-q$. We derive a new recurrence relation for the odd terms of the sequence $\{h(p, q)\}_{n \geqslant 0}$.

Proposition 2.4 The sequence $\left\{h_{2 n+1}\right\}_{n \geqslant 0}$ satisfies the following recurrence relation

$$
h_{2 n+1}=\left(2+p^{2}\right) h_{2 n-1}-\left(1+2 p^{2}-q^{2}\right) h_{2 n-3}+p^{2} \cdot h_{2 n-5},
$$

with $h_{1}=p, h_{3}=p^{3}$ and $h_{5}=p^{5}+p q^{2}$.

Proof: Consider Eq. (10) as a function $f(n)$ in $n$. Computing $f(2 n+1)+p \cdot f(2 n)$ leads to

$$
h_{2 n+1}=\left(1+q+p^{2}\right) h_{2 n-1}-p q \cdot h_{2 n-2}-p^{2} \cdot h_{2 n-3} .
$$

It follows from $f(2 n-1)$ that

$$
p \cdot h_{2 n-4}=-h_{2 n-1}+p \cdot h_{2 n-2}+(1+q) h_{2 n-3} .
$$

Substitute Eq. (13) for the term $p \cdot h_{2 n-4}$ in $p \cdot f(2 n-2)$ and we obtain

$$
p q \cdot h_{2 n-2}=(-1+q) h_{2 n-1}+\left(p^{2}+1-q^{2}\right) h_{2 n-3}-p^{2} \cdot h_{2 n-5} .
$$

The assertion follows from substituting Eq. (14) for the term $p q \cdot h_{2 n-2}$ in Eq. (12).

Now, we are able to prove Theorem 1.2.

Proof of Theorem 1.2. By Eq. (9) and Eq. (11), the sequence $\left\{h_{2 n+1}(p, q)\right\}_{n \geqslant 0}$ shares the same recurrence relation with the sequence $\left\{g_{n}\left(p^{2}, q^{2}\right)\right\}_{n \geqslant 0}$. Their initial conditions are related as follows.

$$
\begin{aligned}
& h_{1}(p, q)=p=p \cdot g_{0}\left(p^{2}, q^{2}\right), \\
& h_{3}(p, q)=p^{3}=p \cdot g_{1}\left(p^{2}, q^{2}\right), \\
& h_{5}(p, q)=p^{5}+p q^{2}=p \cdot g_{2}\left(p^{2}, q^{2}\right) .
\end{aligned}
$$


Suppose the identities $h_{2 k+1}(p, q)=p \cdot g_{k}\left(p^{2}, q^{2}\right)$ hold up to $k=n-1$. Then by induction, we have

$$
\begin{aligned}
h_{2 n+1}(p, q) & =\left(2+p^{2}\right) h_{2 n-1}(p, q)-\left(1+2 p^{2}-q^{2}\right) h_{2 n-3}(p, q)+p^{2} \cdot h_{2 n-5}(p, q) \\
& =\left(2+p^{2}\right) p \cdot g_{n-1}\left(p^{2}, q^{2}\right)-\left(1+2 p^{2}-q^{2}\right) p \cdot g_{n-2}\left(p^{2}, q^{2}\right)+p^{3} \cdot g_{n-3}\left(p^{2}, q^{2}\right) \\
& =p \cdot g_{n}\left(p^{2}, q^{2}\right) .
\end{aligned}
$$

This proved that $b_{2 n+1}(-1, p, q)=p \cdot b_{n}\left(1, p^{2}, q^{2}\right)$ for $n \geqslant 0$. The proof is completed.

For the case of even length, we obtain the following result for the maj-signed fix-desenumerator of $\mathfrak{B}_{2 n}(321)$.

Theorem 2.5 For $n \geqslant 0$, we have

$$
\sum_{\pi \in \mathfrak{B}_{2 n}(321)}(-1)^{\operatorname{maj}(\pi)} p^{f i x(\pi)} q^{\operatorname{des}(\pi)}=\sum_{\pi \in \mathfrak{B}_{n}(321)} p^{2 \cdot f i x(\pi)} q^{2 \cdot \operatorname{des}(\pi)}-q \cdot \sum_{i=0}^{n-1} \sum_{\pi \in \mathfrak{B}_{i}(321)} p^{2 \cdot f i x(\pi)} q^{2 \cdot \operatorname{des}(\pi)} .
$$

Proof: By the same argument as in the proof of Proposition 2.4, we obtain the following recurrence relation for the sequence $\left\{h_{2 n}(p, q)\right\}_{n \geqslant 0}$, which is the same as Eq. (11),

$$
h_{2 n+2}=\left(2+p^{2}\right) h_{2 n}-\left(1+2 p^{2}-q^{2}\right) h_{2 n-2}+p^{2} \cdot h_{2 n-4},
$$

with the initial conditions $h_{0}=1, h_{2}=p^{2}-q$ and $h_{4}=p^{4}-\left(1+p^{2}\right) q+q^{2}$. These polynomials are related to the initial terms of the sequence $\left\{g_{n}\left(p^{2}, q^{2}\right)\right\}_{n \geqslant 0}$ by

$$
\begin{aligned}
& h_{0}(p, q)=1=g_{0}\left(p^{2}, q^{2}\right) \\
& h_{2}(p, q)=p^{2}-q=g_{1}\left(p^{2}, q^{2}\right)-q \cdot g_{0}\left(p^{2}, q^{2}\right) \\
& h_{4}(p, q)=p^{4}-\left(1+p^{2}\right) q+q^{2}=g_{2}\left(p^{2}, q^{2}\right)-q\left(g_{0}\left(p^{2}, q^{2}\right)+g_{1}\left(p^{2}, q^{2}\right)\right) .
\end{aligned}
$$

Making use of the recurrence relation Eq. (9) and Eq. (15) along with the above initial conditions, we can prove the following identity for $n \geqslant 3$ by induction as in the proof of Theorem 1.2

$$
h_{2 n}(p, q)=g_{n}\left(p^{2}, q^{2}\right)-q \cdot \sum_{i=0}^{n-1} g_{i}\left(p^{2}, q^{2}\right)
$$

This proved that for $n \geqslant 0$,

$$
b_{2 n}(-1, p, q)=b_{n}\left(1, p^{2}, q^{2}\right)-q \cdot \sum_{i=0}^{n-1} b_{i}\left(1, p^{2}, q^{2}\right)
$$

The assertion follows. 


\section{Proof of a refined signed-balance identity for $\mathfrak{B}_{n}(123)$}

Note that there is an immediate bijection between $\mathfrak{B}_{n}(321)$ and $\mathfrak{B}_{n}(123)$ by reversion. Though there is no such identity as Eq. (2) for 123-avoiding Baxter Permutations, in this section we shall obtain a maj-des version of refined sign-balance identity for $\mathfrak{B}_{2 n+1}(123)$ based on the results obtained in the previous section.

Corollary 3.1 For all $n \geqslant 0$, we have

$$
\sum_{\pi \in \mathfrak{B}_{2 n+1}(123)}(-1)^{\operatorname{maj}(\pi)} q^{\operatorname{des}(\pi)}=(-1)^{n} q^{2} \cdot \sum_{\pi \in \mathfrak{B}_{n}(123)} q^{2 \cdot \operatorname{des}(\pi)},
$$

where we assume des $(\pi)=-1$ if $\pi$ is an empty word.

Given a permutation $\pi=\pi_{1} \cdots \pi_{n} \in \mathfrak{S}_{n}$, let $\widehat{\pi}=\pi_{n} \cdots \pi_{1} \in \mathfrak{S}_{n}$ be the reverse of $\pi$. For example, if $\pi=21345$ then $\widehat{\pi}=54312$. Since the reverse of a Baxter permutation is also Baxter, the map $\pi \rightarrow \widehat{\pi}$ induces an involution between $\mathfrak{B}_{n}(123)$ and $\mathfrak{B}_{n}(321)$. The major indices and descent numbers of both $\pi$ and $\widehat{\pi}$ satisfy the following relations.

Lemma 3.2 The map $\pi \rightarrow \widehat{\pi}$ induces a bijection between $\mathfrak{B}_{n}(123)$ and $\mathfrak{B}_{n}(321)$ that carries a permutation $\pi \in \mathfrak{B}_{n}(123)$ to $\widehat{\pi} \in \mathfrak{B}_{n}(321)$ with

(i) $\operatorname{des}(\pi)=n-1-\operatorname{des}(\widehat{\pi})$,

(ii) $\operatorname{maj}(\pi)=\left(\begin{array}{l}n \\ 2\end{array}\right)-n \cdot \operatorname{des}(\widehat{\pi})+\operatorname{maj}(\widehat{\pi})$.

Proof: Given a permutation $\pi=\pi_{1} \cdots \pi_{n} \in \mathfrak{B}_{n}(123)$, let $\widehat{\pi}=\widehat{\pi}_{1} \cdots \widehat{\pi}_{n}$, where $\widehat{\pi}_{i}=\pi_{n-i+1}$ for $1 \leqslant i \leqslant n$. Note that $\pi_{i}>\pi_{i+1}$ if and only if $\widehat{\pi}_{n-i}<\widehat{\pi}_{n-i+1}$. Hence $\operatorname{Des}(\pi)=\{i$ : $n-i \notin \operatorname{Des}(\widehat{\pi}), 1 \leqslant i \leqslant n-1\}$. It follows that $\operatorname{des}(\pi)=n-1-\operatorname{des}(\widehat{\pi})$ and

$$
\begin{aligned}
\operatorname{maj}(\pi) & =\sum_{j \in \operatorname{Des}(\pi)} j \\
& =(n-1-\operatorname{des}(\widehat{\pi})) \cdot n-\left(1+\cdots+(n-1)-\sum_{i \in \operatorname{Des}(\widehat{\pi})} i\right) \\
& =\left(\begin{array}{l}
n \\
2
\end{array}\right)-n \cdot \operatorname{des}(\widehat{\pi})+\operatorname{maj}(\widehat{\pi}),
\end{aligned}
$$

The assertion follows.

Consider the generating function $c_{n}(t, q)$ for $\mathfrak{B}_{n}(123)$, respecting the statistics maj and des, defined as

$$
c_{n}(t, q)=\sum_{\pi \in \mathfrak{B}_{n}(123)} t^{\operatorname{maj}(\pi)} q^{\operatorname{des}(\pi)}
$$


and let $C=C(t, q, z)=\sum_{n \geqslant 0} c_{n}(t, q) z^{n}$. For convenience, we assume $\operatorname{des}(\pi)=-1$ if $\pi$ is an empty word, i.e., $c_{0}(t, q)=q^{-1}$ (cf. Lemma 3.2(i) in case $n=0$ ). The initial terms of $C(t, q, z)$ are

$$
\begin{gathered}
C=\frac{1}{q}+z+(1+t q) z^{2}+\left(2\left(t+t^{2}\right) q+t^{3} q^{2}\right) z^{3}+\left(t^{2} q+\left(3 t^{3}+4 t^{4}+3 t^{5}\right) q^{2}+t^{6} q^{3}\right) z^{4} \\
+\left(\left(2 t^{4}+3 t^{5}+2 t^{6}\right) q^{2}+\left(4 t^{6}+6 t^{7}+6 t^{8}+4 t^{9}\right) q^{3}+q^{4} t^{10}\right) z^{5}+\cdots
\end{gathered}
$$

The identity Eq. (17) in Corollary 3.1 is rephrased as

$$
c_{2 n+1}(-1, q)=(-1)^{n} q^{2} \cdot c_{n}\left(1, q^{2}\right) .
$$

For example, for $n=2, c_{2}(t, q)=1+t q$. When $t=1$, we have $c_{2}(1, q)=1+q$. Take

$$
c_{5}(t, q)=\left(2 t^{4}+3 t^{5}+2 t^{6}\right) q^{2}+\left(4 t^{6}+6 t^{7}+6 t^{8}+4 t^{9}\right) q^{3}+q^{4} t^{10} .
$$

When $t=-1$, we have $c_{5}(-1, q)=q^{2}+q^{4}=q^{2} \cdot c_{2}\left(1, q^{2}\right)$. By Lemma 3.2, the polynomial $c_{n}(t, q)$ can be obtained from the polynomial $b_{n}(t, p, q)$ in Eq. (4) with $p=1$. Then the identity Eq. (18) can be derived from the identity Eq. (5).

Proof of Corollary 3.1. By Lemma 3.2, we have

$$
\begin{aligned}
c_{n}(t, q) & =\sum_{\pi \in \mathfrak{B}_{n}(123)} t^{\operatorname{maj}(\pi)} q^{\operatorname{des}(\pi)} \\
& =\sum_{\widehat{\pi} \in \mathfrak{B}_{n}(321)} t^{\left(\begin{array}{c}
n \\
2
\end{array}\right)-n \cdot \operatorname{des}(\widehat{\pi})+\operatorname{maj}(\widehat{\pi})} q^{n-1-\operatorname{des}(\widehat{\pi})} \\
& =t^{\left(\begin{array}{c}
n \\
2
\end{array}\right)} q^{n-1} \cdot b_{n}\left(t, 1, q^{-1} t^{-n}\right) .
\end{aligned}
$$

Then when $t=1$, we have

$$
c_{n}(1, q)=q^{n-1} \cdot b_{n}\left(1,1, q^{-1}\right)
$$

When $t=-1$, we have

$$
\begin{aligned}
& c_{2 n+1}(-1, q)=(-1)^{\left(\begin{array}{c}
2 n+1 \\
2
\end{array}\right)} q^{2 n} \cdot b_{2 n+1}\left(-1,1,-q^{-1}\right) \quad(\text { by Eq. }(19)) \\
& =(-1)^{n} q^{2 n} \cdot b_{n}\left(1,1, q^{-2}\right) \quad \text { (by Eq. (5) with } p=1 \text { ) } \\
& =(-1)^{n} q^{2} \cdot c_{n}\left(1, q^{2}\right) \text { (by Eq. (20)) }
\end{aligned}
$$

The proof is completed.

For the case of even length, we obtain the following result for the maj-signed desenumerator of $\mathfrak{B}_{2 n}(123)$, based on the result in Theorem 2.5.

Corollary 3.3 For all $n \geqslant 0$, we have

$\sum_{\pi \in \mathfrak{B}_{2 n}(123)}(-1)^{\operatorname{maj}(\pi)} q^{\operatorname{des}(\pi)}=(-1)^{n} q \cdot \sum_{\pi \in \mathfrak{B}_{n}(123)} q^{2 \cdot \operatorname{des}(\pi)}-(-1)^{n} \sum_{i=0}^{n-1} q^{2(n-i)}\left(\sum_{\pi \in \mathfrak{B}_{i}(123)} q^{2 \cdot \operatorname{des}(\pi)}\right)$,

where we assume des $(\pi)=-1$ if $\pi$ is an empty word. 
Proof: By Eq. (19) with $t=-1$ and the identities Eq. (16) and (20), we have

$$
\begin{aligned}
& c_{2 n}(-1, q)=(-1)^{\left(\begin{array}{c}
2 n \\
2
\end{array}\right)} q^{2 n-1} \cdot b_{2 n}\left(-1,1, q^{-1}\right) \\
& =(-1)^{n} q^{2 n-1}\left(b_{n}\left(1,1, q^{-2}\right)-q^{-1} \cdot \sum_{i=0}^{n-1} b_{i}\left(1,1, q^{-2}\right)\right) \quad(\text { by Eq. (16) with } p=1) \\
& \left.=(-1)^{n} q \cdot c_{n}\left(1, q^{2}\right)-(-1)^{n} \sum_{i=0}^{n-1} q^{2(n-i)} c_{i}\left(1, q^{2}\right) . \quad \text { (by Eq. }(20)\right)
\end{aligned}
$$

The assertion follows.

\section{Positive braid words on 4 strands and 321-avoiding Baxter permutations}

Recall that the braid group $\mathcal{B}_{3}$ on four strands is generated by $\left\{\sigma_{1}, \sigma_{2}, \sigma_{3}\right\}$ with the relations in Eq. (3). We consider the set $\mathcal{B}_{3}^{+}$of positive braid words with letters $\left\{\sigma_{1}, \sigma_{2}, \sigma_{3}\right\}$. A word $\omega \in \mathcal{B}_{3}^{+}$is called stable if it contains none of the subwords $\left\{\sigma_{3} \sigma_{1}, \sigma_{2} \sigma_{1} \sigma_{2}, \sigma_{3} \sigma_{2} \sigma_{3}\right\}$. Let $\mathcal{W}_{n}$ denote the set of stable words of length $n$ in $\mathcal{B}_{3}^{+}$. For example,

$$
\begin{aligned}
\mathcal{W}_{1}= & \left\{\sigma_{1}, \sigma_{2}, \sigma_{3}\right\} \\
\mathcal{W}_{2}= & \left\{\sigma_{1} \sigma_{1}, \sigma_{1} \sigma_{2}, \sigma_{1} \sigma_{3}, \sigma_{2} \sigma_{1}, \sigma_{2} \sigma_{2}, \sigma_{2} \sigma_{3}, \sigma_{3} \sigma_{2}, \sigma_{3} \sigma_{3}\right\} \\
\mathcal{W}_{3}= & \left\{\sigma_{1} \sigma_{1} \sigma_{1}, \sigma_{1} \sigma_{1} \sigma_{2}, \sigma_{1} \sigma_{1} \sigma_{3}, \sigma_{1} \sigma_{2} \sigma_{1}, \sigma_{1} \sigma_{2} \sigma_{2}, \sigma_{1} \sigma_{2} \sigma_{3}, \sigma_{1} \sigma_{3} \sigma_{2}, \sigma_{1} \sigma_{3} \sigma_{3}, \sigma_{2} \sigma_{1} \sigma_{1},\right. \\
& \left.\sigma_{2} \sigma_{1} \sigma_{3}, \sigma_{2} \sigma_{2} \sigma_{1}, \sigma_{2} \sigma_{2} \sigma_{2}, \sigma_{2} \sigma_{2} \sigma_{3}, \sigma_{2} \sigma_{3} \sigma_{2}, \sigma_{2} \sigma_{3} \sigma_{3}, \sigma_{3} \sigma_{2} \sigma_{1}, \sigma_{3} \sigma_{2} \sigma_{2}, \sigma_{3} \sigma_{3} \sigma_{2}, \sigma_{3} \sigma_{3} \sigma_{3}\right\} .
\end{aligned}
$$

In this section, we establish a bijection between $\mathcal{W}_{n}$ and $\left\{\pi \in \mathfrak{B}_{n+2}(321): \pi^{-1}(1)<\right.$ $\left.\pi^{-1}(2)\right\}$ by constructing a generating tree. A generating tree is a rooted, labeled tree such that the labels of the successors (or children) of each node $v$ can be determined from the label of $v$ itself and a set of succession rules. The notion of generating trees was introduced in [4] for the enumeration of Baxter permutations and further applied to the enumeration of pattern-avoiding permutations (see for instance [12] and [18]).

\subsection{A generating tree for stable positive braid words.}

For a word $\omega=\omega_{1} \cdots \omega_{n} \in \mathcal{W}_{n}$, a prefix of $\omega$ is an initial subword $\omega_{1} \cdots \omega_{i}$ for some $i$ $(1 \leqslant i \leqslant n)$. For $n \geqslant 1$, the words $\omega$ are classified into the following four classes by their prefixes.

- If the first letter of $\omega$ is $\sigma_{1}$, then we label $\omega$ by $\left(2_{1}\right)$.

- If $\omega=\sigma_{2}$ or $\omega$ contains a prefix $\omega^{\prime}$ with $\omega^{\prime} \in\left\{\sigma_{2} \sigma_{1}, \sigma_{2} \sigma_{2}\right\} \cup\left\{\sigma_{3}^{k} \sigma_{2} \sigma_{1}: 2 \leqslant k \leqslant n-2\right\}$, then we label $\omega$ by $\left(3_{1}\right)$.

- If $\omega=\sigma_{3}^{n}$, consisting of $n$ letters of $\sigma_{3}$, then we label $\omega$ by $\left(3_{2}\right)$. 
- Otherwise, we label $\omega$ by $\left(2_{2}\right)$; in this case $\omega$ contains a prefix $\omega^{\prime}$ with $\omega^{\prime} \in\left\{\sigma_{2} \sigma_{3}\right\} \cup$ $\left\{\sigma_{3}^{t} \sigma_{2}: 1 \leqslant t \leqslant n-1\right\}$ but contains none of $\left\{\sigma_{3}^{k} \sigma_{2} \sigma_{1}: 2 \leqslant k \leqslant n-2\right\}$ as prefixes. For example, these words $\sigma_{3} \sigma_{2} \in \mathcal{W}_{2}, \sigma_{3} \sigma_{2} \sigma_{1}, \sigma_{3} \sigma_{2} \sigma_{2} \in \mathcal{W}_{3}$, and $\sigma_{3} \sigma_{3} \sigma_{2} \sigma_{2} \in \mathcal{W}_{4}$ are labeled by $\left(2_{2}\right)$.

For $n \geqslant 0$, the nodes at level $n$ of the generating tree are indexed by the stable words $\omega \in \mathcal{W}_{n}$. The successors $\mu \in \mathcal{W}_{n+1}$ of $\omega$ are obtained by attaching a letter $\sigma_{i}$ at the beginning of $\omega$. If $\mu$ is unstable, then it will be changed into a stable word by replacing a prefix of a certain length, following some rules of replacement.

Proposition 4.1 The generating tree for $\mathcal{W}_{n}$ is given by

$$
\begin{array}{llll}
\text { root } & \left(3_{0}\right) & & \\
\text { rule } & \left(3_{0}\right) & \rightsquigarrow & \left(2_{1}\right)\left(3_{1}\right)\left(3_{2}\right) \\
& \left(2_{1}\right) & \rightsquigarrow & \left(2_{1}\right)\left(3_{1}\right) \\
& \left(2_{2}\right) & \rightsquigarrow & \left(2_{1}\right)\left(2_{2}\right) \\
& \left(3_{1}\right) & \rightsquigarrow & \left(2_{1}\right)\left(3_{1}\right)\left(2_{2}\right) \\
& \left(3_{2}\right) & \rightsquigarrow & \left(2_{1}\right)\left(2_{2}\right)\left(3_{2}\right) .
\end{array}
$$

Proof: The root is the empty word. The successors of the root are the words $\sigma_{1}, \sigma_{2}$, and $\sigma_{3}$, which are labeled by $\left(2_{1}\right),\left(3_{1}\right)$, and $\left(3_{2}\right)$, respectively. For $n \geqslant 1$ and for a word $\omega=\omega_{1} \cdots \omega_{n} \in \mathcal{W}_{n}$, we construct the successors of $\omega$ as follows.

When $\omega$ is labeled by $\left(2_{1}\right)$ it has two successors: $\sigma_{1} \omega$ and $\sigma_{2} \omega$; they are labeled by $\left(2_{1}\right)$ and $\left(3_{1}\right)$, respectively. For the latter word $\mu=\sigma_{2} \omega$, if $\omega_{1} \omega_{2}=\sigma_{1} \sigma_{2}$ then $\mu$ is unstable. We replace the prefix $\sigma_{2} \sigma_{1} \sigma_{2}$ of $\mu$ by $\sigma_{3} \sigma_{3} \sigma_{2}$ if $\omega_{3}=\sigma_{1}$, and by $\sigma_{2} \sigma_{2} \sigma_{3}$ otherwise.

When $\omega$ is labeled by $\left(3_{1}\right)$ it has three successors. There are two cases. If $\omega_{1} \omega_{2} \in$ $\left\{\sigma_{2} \sigma_{1}, \sigma_{2} \sigma_{2}\right\}$, then the successors are $\sigma_{1} \omega, \sigma_{2} \omega$, and $\sigma_{3} \omega$; they are labeled by $\left(2_{1}\right),\left(3_{1}\right)$, and $\left(2_{2}\right)$, respectively. Otherwise, $\omega$ contains a prefix in $\left\{\sigma_{3}^{k} \sigma_{2} \sigma_{1}: 2 \leqslant k \leqslant n-2\right\}$, then the successors are $\sigma_{1} \omega, \sigma_{3} \omega$, and $\sigma_{2} \omega$; they are labeled by $\left(2_{1}\right),\left(3_{1}\right)$, and $\left(2_{2}\right)$, respectively.

When $\omega$ is labeled by $\left(3_{2}\right)$ then $\omega=\sigma_{3}^{n}$ and it has three successors: $\sigma_{1} \omega, \sigma_{2} \omega$, and $\sigma_{3} \omega$; they are labeled by $\left(2_{1}\right),\left(2_{2}\right)$, and $\left(3_{2}\right)$, respectively.

When $\omega$ is labeled by $\left(2_{2}\right)$ it has two successors. There are two cases.

(i) $\omega_{1} \omega_{2}=\sigma_{2} \sigma_{3}$. Then the successors are $\sigma_{1} \omega$ and $\sigma_{3} \omega$; they are labeled by $\left(2_{1}\right)$ and $\left(2_{2}\right)$, respectively. Since the latter word $\mu=\sigma_{3} \omega$ is unstable, a replacement is made as follows. Let $j$ be the least integer such that $\omega_{2}=\cdots=\omega_{j}=3$ and $\omega_{j+1} \neq 3$ for $2 \leqslant j \leqslant n$. Note that $\omega_{j+1}=2$ if $j<n$. Then we assign $\mu=$ $\sigma_{3} \omega_{2} \cdots \omega_{j} \omega_{1} \omega_{j+1} \cdots \omega_{n}=\sigma_{3}^{j} \sigma_{2} \omega_{j+1} \cdots \omega_{n}$.

(ii) $\omega$ contains a prefix in $\left\{\sigma_{3}^{t} \sigma_{2}: 1 \leqslant t \leqslant n-1\right\}$ but contains none of the words in $\left\{\sigma_{3}^{k} \sigma_{2} \sigma_{1}: 2 \leqslant k \leqslant n-2\right\}$ as prefixes. Then the successors are $\sigma_{1} \omega$ and $\sigma_{2} \omega$; they are labeled by $\left(2_{1}\right)$ and $\left(2_{2}\right)$, respectively. 
On the other hand, for $n \geqslant 2$ and for a word $\omega \in \mathcal{W}_{n}$, we shall show that $\omega$ can be uniquely obtained from a word $\tau \in \mathcal{W}_{n-1}$ by the reverse transformations.

Case I. $\omega_{1}=\sigma_{1}$. Then $\omega$ is labeled by $\left(2_{1}\right)$, and the parent of $\omega$ is the word $\tau=$ $\omega_{2} \cdots \omega_{n}$.

Case II. $\omega_{1}=\sigma_{2}$. Consider the second letter of $\omega$. If $\omega_{2}=\sigma_{1}$, then $\omega$ is labeled by $\left(3_{1}\right)$, and the parent of $\omega$ is the word $\tau=\omega_{2} \cdots \omega_{n}$. If $\omega_{2}=\sigma_{3}$, then $\omega$ is labeled by $\left(2_{2}\right)$, and the parent of $\omega$ is the word $\tau=\omega_{2} \cdots \omega_{n}$. Otherwise, $\omega_{2}=\sigma_{2}$ and $\omega$ is labeled by $\left(3_{1}\right)$. The parent of $\omega$ is the word $\tau=\omega_{2} \cdots \omega_{n}$ if $\omega_{3} \in\left\{\sigma_{1}, \sigma_{2}\right\}$, and $\tau=\sigma_{1} \sigma_{2} \omega_{4} \cdots \omega_{n}$ if $\omega_{3}=\sigma_{3}$.

Case III. $\omega_{1}=\sigma_{3}$. There are three subcases.

(a) $\omega=\sigma_{3}^{n}$. Then $\omega$ is labeled by $\left(3_{2}\right)$, and the parent of $\omega$ is the word $\tau=\sigma_{3}^{n-1}$.

(b) $\omega$ contains a prefix in $\left\{\sigma_{3}^{k} \sigma_{2} \sigma_{1}: 2 \leqslant k \leqslant n-2\right\}$. Then $\omega$ is labeled by $\left(3_{1}\right)$. The parent of $\omega$ is $\tau=\omega_{2} \cdots \omega_{n}$ if $k \geqslant 3$, and $\tau=\sigma_{1} \sigma_{2} \sigma_{1} \omega_{4} \cdots \omega_{n}$ if $k=2$.

(c) $\omega$ contains a prefix in $\left\{\sigma_{3}^{t} \sigma_{2}: 1 \leqslant t \leqslant n-1\right\}$ but contains none of words in $\left\{\sigma_{3}^{k} \sigma_{2} \sigma_{1}: 2 \leqslant k \leqslant n-2\right\}$ as prefixes. Then $\omega$ is labeled by $\left(2_{2}\right)$. Factorize $\omega$ as $\omega=\sigma_{3}^{t} \sigma_{2} \omega^{\prime}$. Then the parent of $\omega$ is $\tau=\sigma_{2} \sigma_{3}^{t-1} \omega^{\prime}$.

Note that the label of $\tau$ can be determined by the prefixes of $\tau$, according to the above classification.

The first three levels of the generating tree for $\mathcal{W}_{n}$ are shown in Figure 1, where the label of a word $\omega$ is distinguished by the edge that connects $\omega$ and its parent.

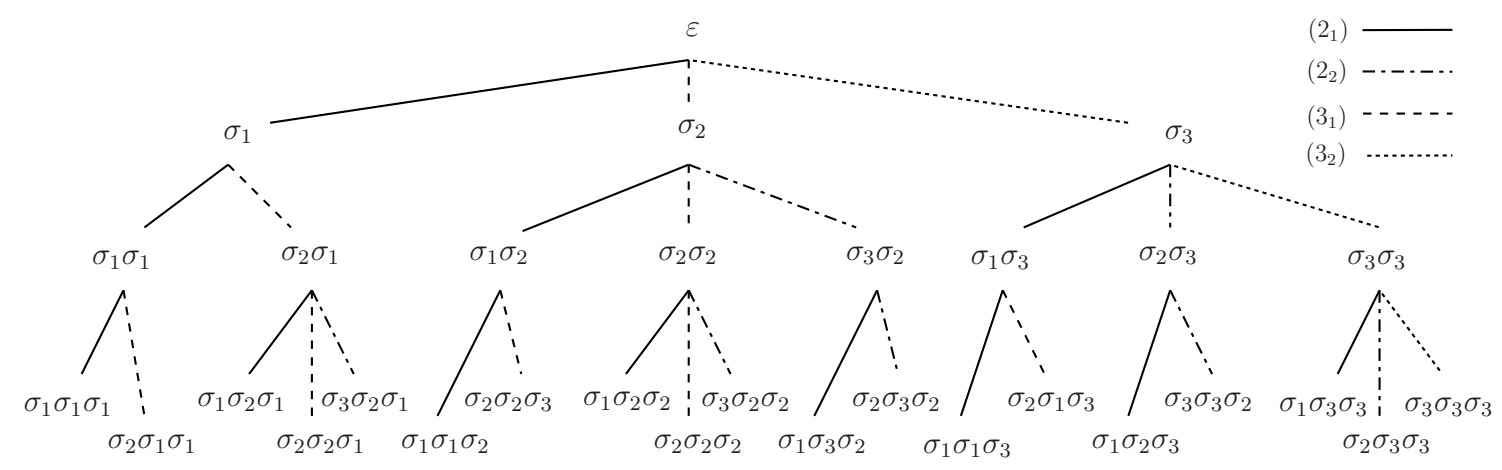

Figure 1: A generating tree for positive braid words on four strands.

Remarks. The transfer matrix of the generating tree for $\mathcal{W}_{n}$ is

$$
A=\left[\begin{array}{lllll}
0 & 1 & 0 & 1 & 1 \\
0 & 1 & 0 & 1 & 0 \\
0 & 1 & 1 & 0 & 0 \\
0 & 1 & 1 & 1 & 0 \\
0 & 1 & 1 & 0 & 1
\end{array}\right]
$$


By a result in [5], we derive the generating function for the size of $\mathcal{W}_{n}$ anew by

$$
u(I-z A)^{-1} v^{T}=\frac{1+z^{2}}{1-3 z+2 z^{2}-z^{3}},
$$

where $u$ is the row vector $(1,0,0,0,0)$ and $v^{T}$ is the column vector $(1,1,1,1,1)^{T}$.

\subsection{1-avoiding Baxter permutations with further restriction}

We consider the permutations in $\mathfrak{B}_{n}(321)$ with the entry 1 preceding the entry 2 . Let

$$
\mathcal{R}_{n}=\left\{\pi \in \mathfrak{B}_{n}(321): \pi^{-1}(1)<\pi^{-1}(2)\right\} .
$$

For example, $\mathcal{R}_{3}=\{123,132,312\}$ and $\mathcal{R}_{4}=\{1234,1243,1324,1342,1423,3124,3412,4123\}$. For $n \geqslant 3$, we classify the permutations $\pi=\pi_{1} \cdots \pi_{n} \in \mathcal{R}_{n}$ into the following four classes.

- If $\pi_{n}=n$ then we label $\pi$ by $\left(2_{1}\right)$.

- If $\pi_{n-1}=n$ then we label $\pi$ by $\left(3_{1}\right)$.

- If $\pi=(3,4, \ldots, n, 1,2)$ then we label $\pi$ by $\left(3_{2}\right)$.

- Otherwise, we label $\pi$ by $\left(2_{2}\right)$.

The generating tree for the set $\mathcal{R}_{n}, n \geqslant 2$, is constructed as follows.

Proposition 4.2 The generating tree for $\mathcal{R}_{n}(n \geqslant 2)$ is given by

$$
\begin{array}{llll}
\text { root } & \left(3_{0}\right) & & \\
\text { rule } & \left(3_{0}\right) & \rightsquigarrow & \left(2_{1}\right)\left(3_{1}\right)\left(3_{2}\right) \\
& \left(2_{1}\right) & \rightsquigarrow\left(2_{1}\right)\left(3_{1}\right) \\
\left(2_{2}\right) & \rightsquigarrow\left(2_{1}\right)\left(2_{2}\right) \\
\left(3_{1}\right) & \rightsquigarrow\left(2_{1}\right)\left(3_{1}\right)\left(2_{2}\right) \\
& \left(3_{2}\right) \rightsquigarrow\left(2_{1}\right)\left(3_{2}\right)\left(2_{2}\right) .
\end{array}
$$

Proof: The root of the generating tree is the permutation $\pi=(1,2)$, and it has three successors $(1,2,3),(1,3,2)$, and $(3,1,2)$; they are labeled by $\left(2_{1}\right),\left(3_{1}\right)$, and $\left(3_{2}\right)$, respectively. For $n \geqslant 3$ and for a $\pi=\pi_{1} \cdots \pi_{n} \in \mathcal{R}_{n}$, we construct the successors of $\pi$ as follows.

If $\pi$ is labeled by $\left(2_{1}\right)$ then it has two successors $(\pi, n+1)$ and $\left(\pi_{1}, \ldots, \pi_{n-1}, n+1, n\right)$; they are labeled by $\left(2_{1}\right)$ and $\left(3_{1}\right)$, respectively.

If $\pi$ is labeled by $\left(3_{1}\right)$ then it is of the form $\left(\pi^{\prime}, i+1, \ldots, n, i\right), i>1$, and has three successors $(\pi, n+1),\left(\pi^{\prime}, i+1, \cdots, n, n+1, i\right)$, and $\left(\pi^{\prime}, i+2, \cdots, n, n+1, i, i+1\right)$; they are labeled by $\left(2_{1}\right),\left(3_{1}\right)$, and $\left(2_{2}\right)$, respectively.

If $\pi$ is labeled by $\left(3_{2}\right)$ then $\pi=(3,4, \ldots, n, 1,2)$ and it has three successors $(\pi, n+1)$, $(3,4, \ldots, n, n+1,1,2)$, and $(4, \ldots, n, n+1,1,2,3)$; they are labeled by $\left(2_{1}\right),\left(3_{2}\right)$, and $\left(2_{2}\right)$, respectively. 
If $\pi$ is labeled by $\left(2_{2}\right)$ then it is of the form $\pi=\left(\pi^{\prime}, i+1, i+2, \ldots, n, j, j+1, \ldots, i\right)$, $i>j$, and has two successors $(\pi, n+1)$ and $\left(\pi^{\prime}, i+2, \ldots, n, n+1, j, j+1, \ldots, i+1\right)$; they are labeled by $\left(2_{1}\right)$ and $\left(2_{2}\right)$, respectively. See Figure 2 for the first three levels of the generating tree.

On the other hand, one can check that for $n \geqslant 3$ every word in $\mathcal{R}_{n}$ can be uniquely obtained from a word in $\mathcal{R}_{n-1}$ by one of the four transformations mentioned above.

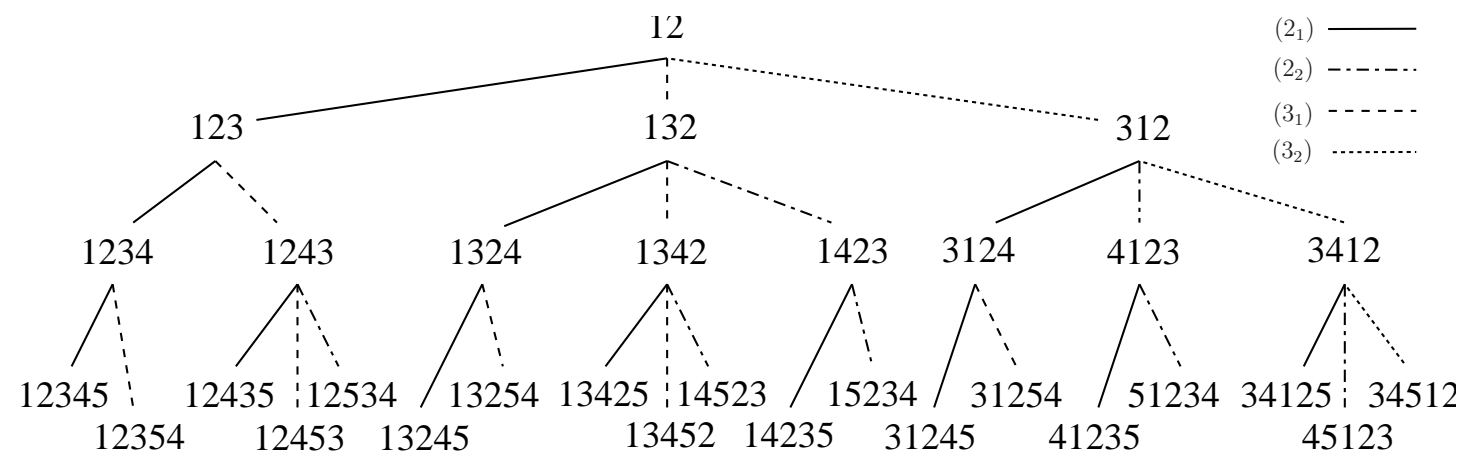

Figure 2: A generating tree for $\pi \in \mathfrak{B}_{n}(321)$ with $\pi^{-1}(1)<\pi^{-1}(2)$.

Proof of Theorem 1.3. By the generating trees in Propositions 4.1 and 4.2, we establish a bijection between $\mathcal{W}_{n}$ and the set $\left\{\pi \in \mathfrak{B}_{n+2}(321): \pi^{-1}(1)<\pi^{-1}(2)\right\}$. This proved Theorem 1.3.

\section{$5 \quad$ Final notes}

As for Theorem 1.2 and Corollary 3.1, our proofs make use of recurrence relations of multivariate generating functions. So far we do not have a combinatorial proof. As shown in [8], there are a number of combinatorial structures bijective with Baxter permutations. We are interested in any connection leading to a combinatorial proof of these results.

Speaking of the phenomenon that the signed enumerator of objects of size $2 n$ is essentially equal to the ordinary enumerator of objects of size $n$, one may ask if the phenomenon holds for other classes of permutations, say $\mathfrak{S}_{n}$, or other statistics. However, this phenomenon seems rare. Note that $\mathfrak{B}_{n}(\omega)=\mathfrak{S}_{n}(\omega)$ for $\omega \in\{132,213,231,312\}$, and there is no such identities for the maj-signed des-enumerators for these $\mathfrak{B}_{n}(\omega)$, or $\mathfrak{B}_{n}$ itself.

Another natural question is that if we restrict the Carlitz's identity to $\mathfrak{B}_{2 n+1}(321)$ is there still a closed form for the generating function

$$
\frac{\sum_{\pi \in \mathfrak{B}_{2 n+1}(321)} t^{\operatorname{maj}(\pi)} q^{\operatorname{des}(\pi)}}{\prod_{i=0}^{n}\left(1-q t^{i}\right)} .
$$

As for the proof of Theorem 1.3, for the purpose of providing recursive bijection with $\mathcal{R}_{n}$, the generating tree for $\mathcal{W}_{n}$ presented in Proposition 4.1 is somewhat artificially 
constructed, with complicated pattern-combinations for nodes and word-replacements for successors. In fact, the generating tree for a class of combinatorial objects is not unique. As pointed out by a referee, another choice of the generating tree for $\mathcal{W}_{n}$ is

$$
\begin{array}{llll}
\omega=\varepsilon \text { (root) } & \left(3_{0}\right) & \rightsquigarrow & \left(2_{1}\right)\left(3_{1}\right)\left(3_{2}\right) \\
\omega=\sigma_{1} \sigma_{2} * & (1) & \rightsquigarrow & \left(2_{1}\right) \\
\omega=\sigma_{1}, \sigma_{1} \sigma_{1} *, \sigma_{1} \sigma_{3} * & \left(2_{1}\right) & \rightsquigarrow & \left(2_{1}\right)\left(3_{1}\right) \\
\omega=\sigma_{2} \sigma_{3} * & \left(2_{2}\right) & \rightsquigarrow & (1)\left(3_{1}\right) \\
\omega=\sigma_{2}, \sigma_{2} \sigma_{1} *, \sigma_{2} \sigma_{2} * & \left(3_{1}\right) & \rightsquigarrow & (1)\left(3_{1}\right)\left(3_{2}\right) \\
\omega=\sigma_{3} * & \left(3_{2}\right) & \rightsquigarrow & \left(2_{1}\right)\left(2_{2}\right)\left(3_{2}\right),
\end{array}
$$

where the stable words $\omega$ are classified by their prefixes shown in the first column. Hence there must be an isomorphic tree for $\mathcal{R}_{n}$ by a certain classification that follows these succession rules while providing the requested generating function. We leave it to interested readers.

One may also ask if the connection between 321-avoiding Baxter permutations and the positive braid words on four strands can be extended further. To our knowledge, it is so far an isolated property and we do not have any generalization.

\section{Acknowledgements.}

The authors thank the referees for carefully reading the manuscript and providing many helpful suggestions. This research is partially supported by NSC grants 101-2115-M-390004 (S.-P. Eu), 101-2115-M-251-001 (T.-S. Fu), and 101-2115-M-127-001 (Y.-J. Pan).

\section{References}

[1] R.M. Adin, Y. Roichman, Equidistribution and sign-balance on 321-avoiding permutations, Sémin. Loth. Combin. 51 (2004) B51d. arXiv:math.C0/0304429.

[2] M. Bousquet-Mélou, Four classes of pattern-avoiding permutations under one roof: generating trees with two labels, Electronic J. Combin. 9 (2003), \#R19.

[3] L. Carlitz, A combinatorial property of q-Eulerian numbers, Amer. Math. Monthly 82 (1975) 51-54.

[4] F. Chung, R. Graham, V. Hoggatt, M. Kleiman, The number of Baxter permutations, J. Combin. Theory Ser. A 24 (1978) 382-394.

[5] E. Deutsch, L. Ferrari, S. Rinaldi, Production matrices, Adv. Appl. Math. 34 (2005) $101-122$.

[6] S. Dulucq, O. Guibert, Baxter permutations, Discrete Math. 180 (1998) 143-156.

[7] S.-P. Eu, T.-S. Fu, Y.-J. Pan, C.-T. Ting, Sign-balance identities of Adin-Roichman type on 321-avoiding alternating permutations, Discrete Math. 312 (2012) 2228-2237.

[8] S. Felsner, E. Fusy, M. Noy, D. Orden, Bijections for Baxter families and related objects, J. Combin. Theory Ser. A 118 (2011) 993-1020. 
[9] C. Kassel, V. Turaev, Braid Groups, GTM 247, Springer, New York 2008.

[10] C.L. Mallows, Baxter permutations rise again, J. Combin. Theory Ser. A 27 (1979) 394-396.

[11] T. Mansour, Equidistribution and sign-balance on 132-avoiding permutations, Sémin. Loth. Combin. 51 (2004) B51e.

[12] T. Mansour, V. Vajnovszki, Restricted 123-avoiding Baxter permutations and the Padovan numbers, Discrete Appl. Math. 155 (2007) 1430-1440.

[13] A. Reifegerste, Refined sign-balance on 321-avoiding permutations, European J. Combin. 26 (2005) 1009-1018.

[14] R. Simion, F.W. Schmidt, Restricted permutations, European J. Combin. 6 (1985) 383-406.

[15] N.J.A. Sloane, The On-Line Encyclopedia of Integer Sequences, http://oeisf . org/.

[16] R. Stanley. Some remarks on sign-balanced and maj-balanced posets. Adv. Appl. Math. 34(4) (2005) 880-902.

[17] G. Viennot, A bijective proof for the number of Baxter permutations, Sémin. Loth. Combin., Le Klebach, 1981.

[18] J. West, Generating trees and the Catalan and Schröder numbers, Discrete Math. 146 (1995) 247-262. 